\title{
Influence of 3D Effects on Field Quality in the Straight Part of Accelerator Magnets for the High Luminosity Large Hadron Collider
}

\author{
E. Nilsson, S. Izquierdo Bermudez, E. Todesco, S. Enomoto, S. Farinon, P. Fabbricatore, \\ T. Nakamoto, M. Sugano and F. Savary.
}

\begin{abstract}
A dedicated D1 beam separation dipole is currently being developed at KEK for the Large Hadron Collider Luminosity upgrade (HL-LHC). Four $150 \mathrm{~mm}$ aperture, $5.6 \mathrm{~T}$ magnetic field and $6.7 \mathrm{~m}$ long $\mathrm{Nb}$ - $\mathrm{Ti}$ magnets will replace resistive $\mathrm{D} 1$ dipoles. The development includes fabrication and testing of $\mathbf{2 . 2}$ m model magnets. The dipole has a single layer coil and thin spacers between coil and iron, giving a non-negligible impact of saturation on field quality at nominal field. The magnetic design of the straight section coil cross section is based on 2D optimization and a separate optimization concerns the coil ends. However, magnetic measurements of the short model showed a large difference (tens of units) between the sextupole harmonic in the straight part and the 2D calculation. This difference is correctly modelled only by a 3D analysis: 3D calculations show that the magnetic field quality in the straight part is influenced by the coil ends, even for the $6.7 \mathrm{~m}$ long magnets. The effect is even more remarkable in the short model. We investigate similar 3D effects for other magnets, namely the $11 \mathrm{~T}$ dipole for HL-LHC. We also consider the case of the 4.5 T recombination magnets for HL-LHC (D2), where the larger space between coil and iron makes this effect less important, but still visible. We conclude the paper by outlining the different classes of accelerator magnets where this coupling between $3 D$ effects and iron saturation can be relevant.
\end{abstract}

Index Terms - Superconducting magnets, accelerator magnets, magnetic analysis.

\section{INTRODUCTION}

$\mathrm{I}_{\mathrm{C}}^{\mathrm{N}}$ PARTICLE ACCELERATORS such as the Large Hadron Collider (LHC) the particle beam is guided in its circular orbit by the magnetic field generated by dipole magnets. Requirements on the magnetic field are extremely high as field errors can be detrimental for the beam dynamics. As a first approach the magnetic design of superconducting accelerator magnets is usually based on numerical optimization by 2D modelling of the cross section in the transverse plane [1]. Once the cross section is designed, the coil ends are optimized separately with the objective to minimize the total field errors

Automatically generated dates of receipt and acceptance will be placed here. E. Nilsson, S. Izquierdo Bermudez, E. Todesco and F. Savary are with the European Organization for Nuclear Research, CERN, 1211 Geneva, Switzerland (e-mail: emelie.nilsson@cern.ch).

S. Enomoto, T. Nakamoto and M. Sugano are with High Energy Accelerator Research Organization (KEK), Tsukuba 305-0801, Japan.

$\mathrm{S}$. Farinon and P. Fabbricatore are with Instituto Nazionale di Fisica Nucleare Sezione di Genova, 16146 Genova, Italy. integrated along the magnet length. The main accelerator magnets in the LHC typically have a length of the order of meters, and clear aperture radius of less than $0.1 \mathrm{~m}$. Until now, these magnets were typically considered long enough for a 2D model to be a valid description of the field in the straight part. However, recent magnetic measurements on the $2.2 \mathrm{~m} \mathrm{D} 1$ separation dipole indicate that field contribution from the extremities reach into the center and strongly modify the field calculated with the $2 \mathrm{D}$ model [2]. In this paper we show that for some classes of dipoles the magnetic field quality in the straight part is significantly affected by the coil ends and a 3D analysis is required.

We focus on 3D modelling of four superconducting dipole magnets currently developed for the High Luminosity upgrade of the LHC (HL-LHC): the D1, D2 and the $11 \mathrm{~T}$ dipole in single and twin aperture [3]. The main parameters of the magnets are summarized in Table I along with cross sections in Fig. 1. The dipoles are rather different in terms of field, aperture and layout, which makes them interesting to compare to each other in this study.

Resistive D1 separation dipoles are being replaced by superconducting $5.6 \mathrm{~T}$, single layer $\mathrm{Nb}$ - $\mathrm{Ti}$ magnets with $150 \mathrm{~mm}$ aperture [4]. The design study and development of the $2.2 \mathrm{~m}$ short model is currently ongoing in collaboration between CERN and KEK. It is wound from spare $\mathrm{Nb}$-Ti cable of the outer layer conductor of the LHC main dipoles, in a single layer in order to maximize the cooling and allow for a larger yoke, which reduces fringe fields. In Sec. II two independent numerical models are presented to explain the origin of the tens of additional sextupole units seen on the magnetic measurements of the D1 short model magnet. The $3 \mathrm{D}$ model predicts that even the full length $6.7 \mathrm{~m}$ D1 magnet is affected by the coil end effects, but less severely.

TABLE I. MAIN PARAMETERS OF THE DIPOLES CONSIDERED IN THIS WORK.

\begin{tabular}{lcccc}
\hline \hline & 11T 1in1 & 11T 2in1 & D1 & D2 \\
\hline Bore field at $\mathbf{I}_{\text {nom }}(\mathbf{T})$ & 11.2 & 11.2 & 5.6 & 4.5 \\
Nr. of apertures & 1 & 2 & 1 & 2 \\
Beam separation (mm) & -- & 194 & -- & 188 \\
Aperture radius (mm) & 30 & 30 & 75 & 53 \\
Coil width (mm) & 31 & 31 & 15 & 15 \\
Distance coil to yoke (mm) & 31 & 31 & 21 & 36 \\
Yoke inner radius (mm) & 92 & -- & 111 & -- \\
Yoke outer radius (mm) & 255 & 275 & 275 & 307 \\
Short model magnetic length (m) & 1.7 & 1.7 & 1.7 & 1.4 \\
Full magnet magnetic length (m) & 5.3 & 5.3 & 6.3 & 7.8 \\
\hline
\end{tabular}


a) D1

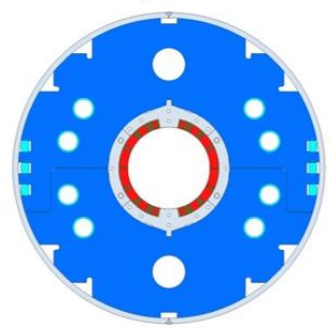

b) D2

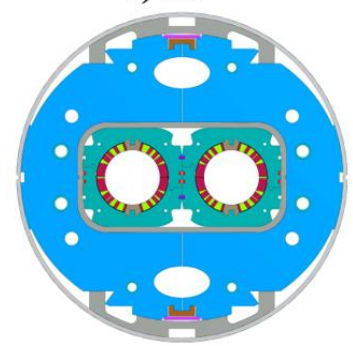

c) $11 \mathrm{~T}$ single aperture

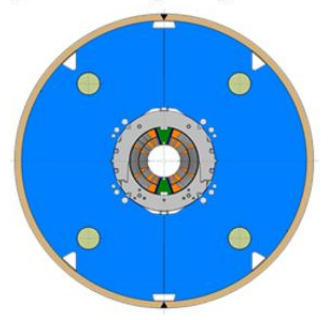

d) $11 \mathrm{~T}$ twin aperture

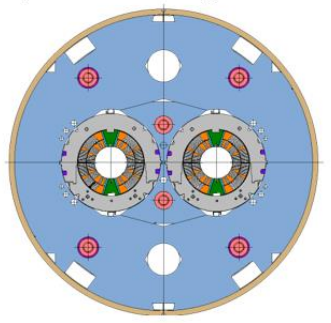

Fig. 1. Cross section layouts of the (a) D1 separation dipole, (b) D2 recombination dipole, and the $11 \mathrm{~T}$ dipole in single (c) and double (d) aperture.

These findings are compared in Sec. III to the $11 \mathrm{~T}$ dipole in its single and double aperture. The $5.6 \mathrm{~m}$ long $11 \mathrm{~T} \mathrm{Nb}_{3} \mathrm{Sn}$ magnets will replace some of the $8.3 \mathrm{~T} 15 \mathrm{~m}$ long Nb-Ti LHC main dipoles to clear longitudinal space for additional collimators. The $2 \mathrm{~m}$ short models are tested in single and double aperture configuration.

The final magnet studied is the double aperture, $\mathrm{Nb}-\mathrm{Ti}$, 4.5 T D2 recombination dipole [5]. Its fields are directed in the same direction in both apertures, as opposed to the $11 \mathrm{~T}$ dipole. In Sec. IV the 3D effects of the two types of field configurations are compared.

In Sec. $\mathrm{V}$ the impact of critical design parameters on 3D effects is explored, with the aim to identify layouts for which coil end effects are minimized and to learn why certain dipole magnets are more sensitive to these $3 \mathrm{D}$ effects.

\section{THE D1 SEPARATION DIPOLE}

\section{A. Modelling of the short model D1 magnet}

Normal $\left(b_{n}\right)$ and skew $\left(a_{n}\right)$ components of the field harmonic expansion are defined as

$B(x, y)=\sum_{n=1}^{\infty}\left(B_{n}+i A_{n}\right)\left(\frac{x+i y}{R_{r e f}}\right)^{n-1}=10^{-4} B_{1} \sum_{n=1}^{\infty}\left(b_{n}+i a_{n}\right)\left(\frac{x+i y}{R_{r e f}}\right)^{n-1}$,

where $B_{1}$ is the main field and $\mathrm{R}_{\mathrm{ref}}$ is the reference radius typically defined as $2 / 3$ of the aperture radius. Integrated multipoles over the longitudinal direction $z$ are expressed in

TABLE II. MAGNETIC FIELD COMPARISON OF THE D1 SHORT MODEL CALCULATED IN ROXIE 2D AND 3D AT NOMINAL CURRENT (12 KA) AT $\mathrm{R}_{\mathrm{REF}}=50 \mathrm{MM}$, INCLUDING THE KEK IRON PIT.

\begin{tabular}{ccccccc}
\hline \hline ROXIE at $\mathrm{I}_{\text {nom }}$ & \multicolumn{2}{c}{$2 \mathrm{D}$} & \multicolumn{3}{c}{$3 \mathrm{D}$} & \multicolumn{2}{c}{ Diff 2D $\rightarrow$ 3D } \\
units at $\mathrm{R}_{\mathrm{ref}}=50 \mathrm{~mm}$ & $\mathrm{~b}_{3}$ & $\mathrm{~b}_{5}$ & $\mathrm{~b}_{3}$ & $\mathrm{~b}_{5}$ & $\Delta \mathrm{b}_{3}$ & $\Delta \mathrm{b}_{5}$ \\
\hline Coil only & -32.2 & 8.6 & -32.4 & 8.4 & -0.3 & -0.2 \\
\hline Simplified yoke & 1.8 & -2.4 & 28.1 & -0.1 & 26.4 & 2.3 \\
\hline Full yoke geometry & -1.3 & -2.0 & -- & -- & -- & -- \\
\hline
\end{tabular}

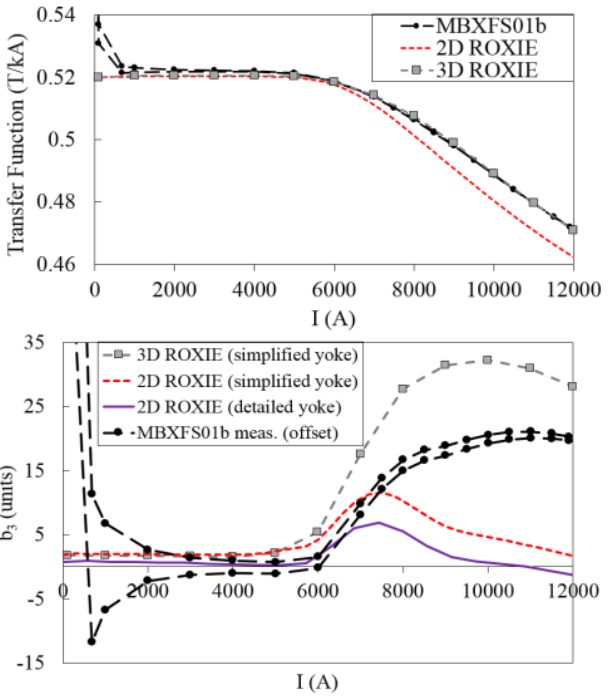

Fig. 2. Comparison of $2 \mathrm{D}$ and $3 \mathrm{D}$ calculations of the D1 dipole. Different saturation behavior is found on the transfer function (top) and the sextupole component (bottom).

units $\left(10^{-4}\right)$ of the main integrated dipole component:

$$
\bar{b}_{n}=\frac{\int_{-\infty}^{+\infty} B_{n}(I, z) d z}{B_{1}^{s s}(I) l_{\text {mag }}(I)},
$$

where $B_{I} S S$ is the main field in the straight section and $l_{\text {mag }}$ is the magnetic length and $I$ is the current:

$$
l_{\text {mag }}(I)=\frac{1}{B_{1}^{s s}(I)} \int_{-\infty}^{+\infty} B_{1}(I, z) d z \text {. }
$$

The different saturation behavior between $2 \mathrm{D}$ and $3 \mathrm{D}$ was already noticed in the design phase of the full length $6.7 \mathrm{~m}$ magnet, where $b_{3}$ in the center exceeds the $2 \mathrm{D}$ value by around one unit and $0.02 \mathrm{~T}$ for the main field at the reference radius $R_{\text {ref }}=50 \mathrm{~mm}$ [6]. In the short model the effect of the ends is far more significant: around 30 additional units are predicted in the center of the magnet. If the iron yoke is excluded in the model, the $3 \mathrm{D}$ effect is negligible and $\mathrm{b}_{3}$ is constant along the longitudinal axis in the straight section. However, with the iron yoke there is a strong, long-range disturbance from the magnet ends, and even though $b_{3}$ decreases towards the magnet center, it is around 30 units higher than the 2D result (Fig. 2). Magnetic measurements are closer to the $3 \mathrm{D}$ than $2 \mathrm{D}$ predictions. Lower iron saturation is clearly seen on the transfer function (Fig. 1), where additional 180 units, or $0.08 \mathrm{~T}$, are found at nominal current for the 3D model. The magnetic field calculations done with ROXIE [7] are summarized in Table I. The KEK iron pit, different from the LHC cryostat, is also included in the model. A simplified iron geometry is used for the calculations, to ease convergence

TABLE III. THE D1 SHORT MODEL MAGNETIC FIELD MODELLED IN OPERA AND ROXIE AT NOMINAL CURRENT ( $12 \mathrm{KA})$ AT $\mathrm{R}_{\text {REF }}=50 \mathrm{MM}$. THE RECTANGULAR NOTCH ON THE YOKE INNER DIAMETER AND IRON PIT ARE EXCLUDED.

\begin{tabular}{lcccccc}
\hline \hline & 2 & & & & & \\
& & & & & & \\
& & & & & \\
\hline & $\mathrm{B}_{1}(\mathrm{~T})$ & $\mathrm{b}_{3}$ (units) & \multicolumn{1}{c}{$\mathrm{B}_{1}(\mathrm{~T})$} & $\mathrm{b}_{3}$ (units) & $\mathbf{\Delta B}_{\mathbf{1}}$ & $\boldsymbol{\Delta}_{\mathbf{3}}$ \\
\hline OPERA & 5.556 & -5.9 & 5.683 & 24.5 & $\mathbf{0 . 1 3}$ & $\mathbf{3 0 . 4}$ \\
ROXIE & 5.567 & -6.8 & 5.675 & 21.3 & $\mathbf{0 . 1 1}$ & $\mathbf{2 8 . 1}$ \\
diff. models & -0.01 & 0.8 & 0.01 & 3.2 & -- & -- \\
\hline
\end{tabular}


of the 3D model. This offsets $b_{3}$ by 3 units according to the 2D calculations (Table II).

To benchmark the results from the D1 short model, a separate simulation is set up with the OPERA software from Cobham Vector Fields [8]. The OPERA-2D and -3D software are finite element models (FEM), whereas ROXIE uses a hybrid approach of boundary element model (BEM) and FEM. In the OPERA model the conductor geometry is imported from ROXIE and the same iron yoke nonlinear permeability is used. For a better convergence the rectangular notch at the inner diameter of the iron yoke is excluded, as well as the KEK iron pit (Fig. 3). A close agreement is found between the OPERA and ROXIE models of the $2.2 \mathrm{~m}$ simplified D1 model (Table III). The 1.5 unit offset of $b_{3}$ in the OPERA model is explained by the homogeneous current density in the conductors. In ROXIE, on the other hand, the current density is graded for a more realistic description of the inhomogeneous current density in keystoned cables. Applying also constant current density in the ROXIE 2D model results in agreement with the OPERA-2D model of $b_{3}$ within 0.4 units. Although the magnetic measurements are closer to the 3D than 2D model results, there are details at intermediate to high current that remain to be understood.

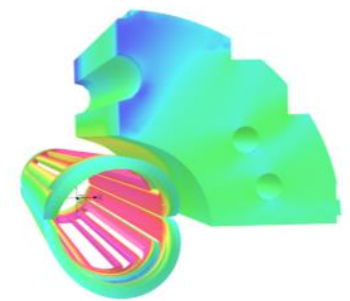

Fig. 3. The OPERA-3D model with simplified iron yoke geometry.

\section{B. Modelling of the full length DI magnet}

The short model magnet is housed in the KEK iron pit, as opposed to the full length magnet, which is inserted in a LHC cryostat. For comparison of the magnetic 3D models of short and full length magnet, only the iron yoke is included in the results presented in this section. Integral field harmonics at nominal current are shown in Table IV. The multipole content differs not only between the short model and full length magnet in the straight section, but also in the magnet ends, which indicates that the extremities in the short model not only affect the straight section, but also the multipole content

TABLE IV. INTEGRATED FIELD HARMONICS AT NOMINAL CURRENT. SAME YOKE GEOMETRY IS USED FOR SHORT AND FULL LENGTH MAGNET (CRYOSTATS EXCLUDED).

\begin{tabular}{ccccccccc}
\hline \hline & \multicolumn{1}{c}{ Straight part } & \multicolumn{3}{c}{ Ends } \\
\hline \multicolumn{1}{c}{} \\
& \multicolumn{1}{c}{ C.S. } & \multicolumn{2}{c}{ N.C.S. } \\
\hline & $\begin{array}{l}\text { short } \\
\text { model }\end{array}$ & $\begin{array}{l}\text { full } \\
\text { length }\end{array}$ & $\begin{array}{l}\text { short } \\
\text { model }\end{array}$ & $\begin{array}{l}\text { full } \\
\text { length }\end{array}$ & $\begin{array}{l}\text { short } \\
\text { model }\end{array}$ & $\begin{array}{l}\text { full } \\
\text { length }\end{array}$ & $\begin{array}{l}\text { short } \\
\text { model }\end{array}$ & $\begin{array}{l}\text { full } \\
\text { length }\end{array}$ \\
\hline $\begin{array}{l}\mathbf{L}_{\mathbf{m}} \\
(\mathbf{m})\end{array}$ & 0.1 & 4.7 & 0.8 & 0.8 & 0.8 & 0.8 & 1.7 & 6.3 \\
$\mathbf{b}_{3}$ & 27.2 & 3.1 & 0.6 & -20.0 & -4.9 & -26.6 & -0.1 & -3.7 \\
$\mathbf{b}_{5}$ & -0.2 & -2.4 & 0.3 & -1.9 & -3.3 & -5.5 & -1.4 & -2.7 \\
$\mathbf{b}_{7}$ & 0.2 & 0.3 & -1.5 & -1.3 & -3.3 & -3.2 & -2.2 & -0.4 \\
$\mathbf{a}_{1}$ & 0.3 & 0.1 & -34.8 & -30.4 & 0.2 & 0.0 & -16.2 & -4.0 \\
$\mathbf{a}_{3}$ & 0.1 & 0.0 & 12.5 & 11.8 & 0.0 & 0.0 & 5.9 & 1.6 \\
\hline
\end{tabular}

in the opposite magnet end.

Magnet ends compensate the enhanced multipole content in the straight section. The integrated $b_{3}$ in Table IV can be compared to the $2 \mathrm{D}$ calculation for the same geometry $b_{3}=-1.2$ units (Fig. 2). For the short model, including the KEK iron pit, the integrated value is one unit higher than its 2D prediction. For the full length magnet the integrated $b_{3}$ is 2.5 unit lower than for the 2D calculation.

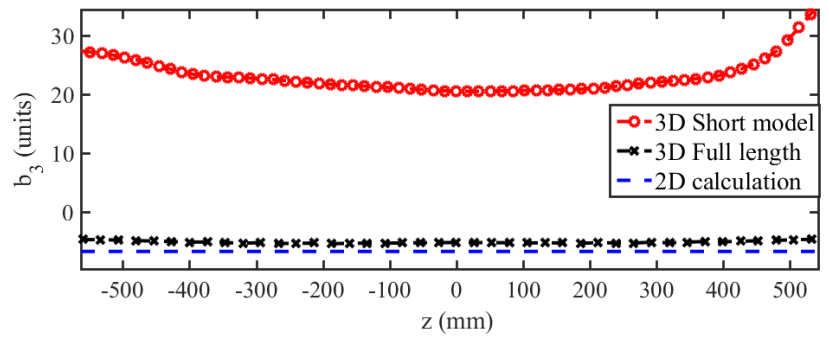

Fig. 4. Comparison of axial distribution, or pseudo multipole, of $b_{3}$ for short and full length D1 magnets. For increased length $b_{3}$ approaches the $2 \mathrm{D}$ result, but additional 1.5 units of $b_{3}$ are found in the magnet center for the full length magnet.

The simplifications of the iron geometry for the 3D calculations add around 3 units on $b_{3}$ at nominal current. Hence the $b_{3}$ values in Table IV should be compensated by -3 units. The KEK iron pit (short model) adds another 3 units in 2D and the LHC cryostat (full length magnet) adds around 8 units in 2D. With all the above accounted for the short model is expected to have integrated $b_{3}$ near 0 and the full length magnet around +1 unit. As the integral field harmonics are within tolerances, re-optimization to correct for global effect of the coil ends is not necessary.

\section{THE 11 T DIPOLE}

The 3D effects for $11 \mathrm{~T}$ are much less significant than for the $\mathrm{D} 1$ dipole, but still visible. The transfer function of the 3D calculation is compared to the measurements of single and double aperture models (Fig. 5). The measurements of the single aperture models MBHSP102 and MBHSP103 show different saturation behavior compared to the $2 \mathrm{D}$ calculation [9]. The discrepancy in the transfer function at nominal current is 70 units. The 3D calculation results in much closer agreement with magnetic measurements. For single aperture 2 additional units on $\mathrm{b}_{3}$ are found in the 3D model compared to 2D. Owing to persistent current effects on the harmonics, the 3D effect has not been validated on magnetic measurements. On the other hand, for the double aperture magnet the iron saturation is well captured in the 2D model (Fig. 3). The double aperture 3D and 2D analysis show no difference in saturation, apart from a constant offset of 15-20 units at all current levels. When the same collared coils used in MBHSP102 and SP103 are assembled in double aperture configuration (MBHDP102), the measurements yield good agreement with the $2 \mathrm{D}$ model. This result indicates that the end effects of the $11 \mathrm{~T}$ influences field quality in the center of the magnet in single aperture, but not in double aperture.

The absence of coil end effects on the double aperture $11 \mathrm{~T}$ dipole could possibly be explained qualitatively by the 
opposite directions of field in the two apertures, which would allow for magnetic connection between the two apertures. This hypothesis can be easily tested by reversing the magnetic field in one of the apertures, such that $B_{y}$ is directed in the same way in both apertures. Indeed, in such a configuration a significant difference is found between the 2D and 3D models. The difference appears at lower current than in the single aperture case, namely at $3 \mathrm{kA}$ and the $3 \mathrm{D}$ model adds $\Delta \mathrm{b}_{3}=3.5$ units at $11.85 \mathrm{kA}$. Such a layout is reminiscent of the D2 dipole, treated in the following section.
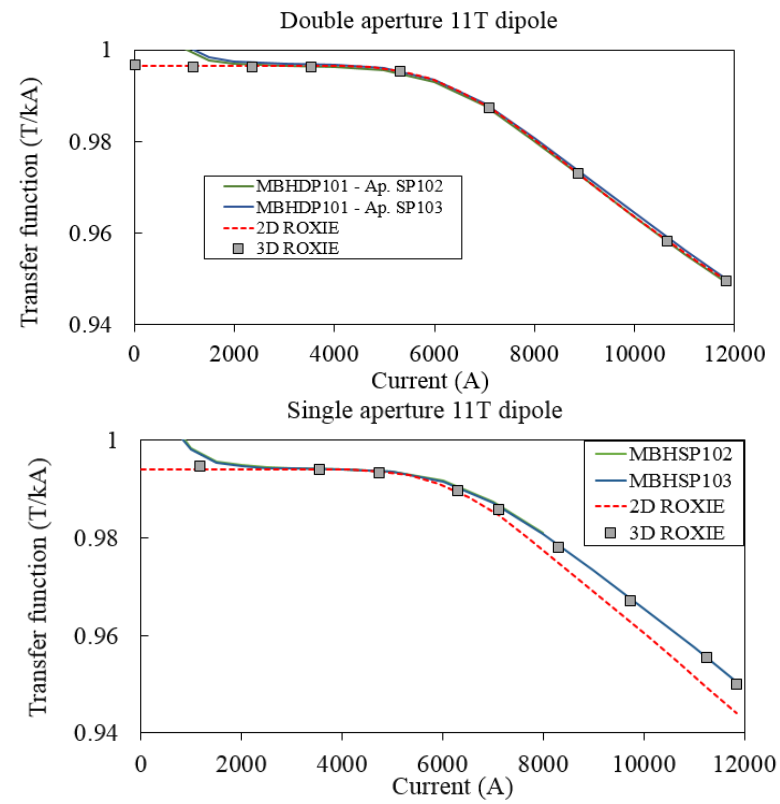

Fig. 5. Good agreement between the ROXIE model and measurements is found for the transfer function of the double aperture $11 \mathrm{~T}$ dipole. For single aperture, there is a significant discrepancy between the 2D and 3D model and measurements are understood only with the $3 \mathrm{D}$ model.

\section{THE D2 RECOMBINATION DIPOLE}

Comparison of its 2D and 3D models shows that 3D effects are visible on the short model but less important, namely $\Delta \mathrm{b}_{3}=1$ unit at nominal current $(12 \mathrm{kA})$. This small correction appears to be a consequence of the large distance between the coil and the iron yoke, as when placing the iron closer to the coil, the 3D-effects grow more significant. For the full length D2 dipole, the coil end effects are negligible in the center.

\section{PARAMETRIC STUDY FOR SINGLE APERTURE DIPOLES}

As shown in Sec. II-IV the field enhancement in the magnet center between 2D and 3D models for single aperture dipoles may range from a few units of $b_{3}(11 \mathrm{~T})$ to tens of units (the D1 short model). The magnet length is a critical parameter, and hence there will be a length at which the magnetic field in the central part of the magnet is unperturbed by the coil ends. As the available longitudinal space in accelerators is tightly constrained, it is useful to explore the impact of design parameters in the transverse plane to understand why the $3 \mathrm{D}$ effect is much more significant for D1 than for $11 \mathrm{~T}$.

Parametric studies show that the 3D effects are strongly dependent on (1) the distance between the coil and yoke, (2) the coil aperture and (3) the area of the yoke cross section. For the first point, increased space between coil and iron yoke strongly reduces the 3D effects. In Fig. 6 the inner radius of the yoke is varied, and the outer radius is adjusted to keep the nominal yoke area for $11 \mathrm{~T}$ and $\mathrm{D} 1$. The parameter scan reveals that the small space between inner yoke radius and coil is the main reason behind the strong 3D effects in D1. Imagining a D1 dipole with the same ratio between coil aperture and yoke inner dimension as in $11 \mathrm{~T}$, the inner yoke radius would be $230 \mathrm{~mm}$, at which the $\Delta \mathrm{b}_{3}$ between $3 \mathrm{D}$ and $2 \mathrm{D}$ model would be one unit rather than tens of units.

Regarding the amount of iron yoke in the 2D cross section, there exists an area for which 3D effects are maximized (Fig. 6). In order to separate the strong effect of the distance between yoke and coil, the inner radius is kept constant and the area is adjusted by modifying only the outer yoke dimension. In the absence of iron yoke $\Delta b_{3}=0$, but adding a small iron yoke the difference increases, up until a critical yoke cross section area at which $\Delta b_{3}$ is maximized. The nominal yoke area of D1 unfortunately maximizes $\Delta \mathrm{b}_{3}$, which combined with the small spacer between coil and yoke explains its strong 3D-effects. The $11 \mathrm{~T}$ dipole on the other hand, has a yoke area beyond the maximum, which is more favorable for reduced $3 \mathrm{D}$ effects.
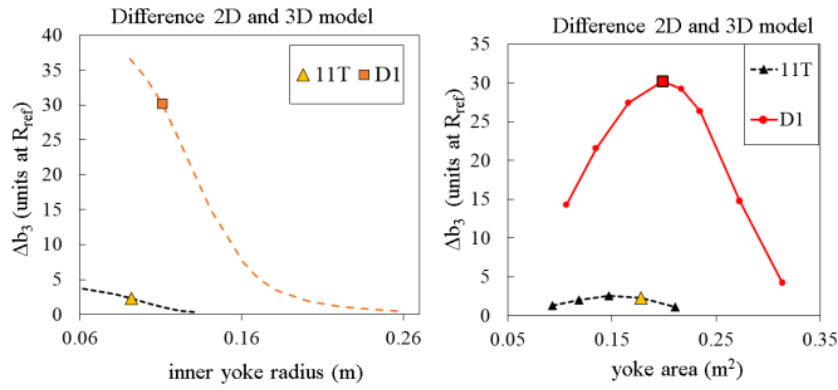

Fig. 6. The difference in $b_{3}$ of $2 \mathrm{D}$ and $3 \mathrm{D}$ model in the magnet center for $\mathrm{D} 1$ and $11 \mathrm{~T}$ (single aperture), as a function of inner yoke radius, for the nominal yoke areas. Reference radius $R_{\text {ref }}$ is $50 \mathrm{~mm}$ and $17 \mathrm{~mm}$ respectively. The difference in $b_{3}$ also varies strongly with the yoke area, given an inner yoke radius. The enlarged square and triangle represents nominal configuration of D1 and 11 T respectively.

\section{CONCLUSION}

The field in the straight part of accelerator magnets requires special attention in certain magnet configurations due to perturbations by its coil ends. In this work the influence of coil end effects on field quality in the straight part of the D1, D2 and $11 \mathrm{~T}$ dipoles is studied. Non-negligible difference between 2D and 3D models are found on the single aperture short models for transfer function and sextupole component, in particular in the D1 dipole where tens of additional units were measured in the magnet center of the $2 \mathrm{~m}$ model. The effect is explained only by 3D modelling. For the single aperture $11 \mathrm{~T}$ dipole a weaker, but still visible effect is found. In double aperture $11 \mathrm{~T}$ the effect is negligible.

Single aperture dipoles are prone to coil end effects, but the effect decreases strongly with the distance between coil and yoke. Parametric studies show the feasibility of optimizing single aperture dipoles in order to minimize the influence of magnet ends on the magnetic field in the straight section. 


\section{REFERENCES}

[1] M. Sugano et al., "Field Quality and Mechanical Analysis of the Beam Separation Dipole for HL-LHC Upgrade", IEEE Trans. Appl. Supercond., vol. 25, no. 3, Jun. 2015, Art. no. 4001105.

[2] S. Enomoto et al., "Field Measurement to evaluate iron saturation and coil end effects in the 2-m Model Magnet of Beam Separation Dipole for the HL-LHC Upgrade", same conference.

[3] The HiLumi LHC Collaboration, HL-LHC Preliminary Design Report, CERN-ACC-2014-0300, 2014.

[4] T. Nakamoto et al., "Model Magnet Development of D1 Beam Separation Dipole for the HL-LHC Upgrade", IEEE Trans. Appl. Supercond., vol. 25, no. 3, Jun. 2015, Art. no. 4000505.

[5] S. Farinon et al., "The Design of Superconducting Separation Dipoles D2 for the High Luminosity Upgrade of LHC", IEEE Trans. Appl. Supercond., vol. 26, no. 4, Jun. 2016, Art. no. 4001504.

[6] Q. Xu et al., "Design Optimization of the New D1 Dipole for HL-LHC Upgrade", IEEE Trans. Appl. Supercond., vol. 24, no. 3, Jun. 2014, Art. no. 4000104.

[7] S. Russenschuck, "ROXIE: A Computer Code for the Integrated Design of Accelerator Magnets", LHC Project Report 276, 1999.

[8] Cobham Vector Fields Opera, http://operafea.com

[9] L. Fiscarelli et al., "Magnetic Analysis of the first 11- $\mathrm{T} \mathrm{Nb}_{3} \mathrm{Sn}$ Dipole Models developed at CERN for HL-LHC", IEEE Trans. Appl.

Supercond., vol. 26, no. 4, Jun. 2016, Art no. 4003805. 